\title{
Emphysematous pyelonephritis, ureteritis, and cystitis: Report of a case
}

\author{
PHILLIP C. GINSBERG, Do \\ PERRY WEINER, DO \\ ROBERT KLAUS, MD \\ WILLIAM HERRING, MD \\ SCOT E. GOLDBERG, DO
}

\begin{abstract}
Emphysematous
pyelonephritis is a rare infection of the genitourinary tract. It is easily recognized radiographically because of the characteristic gas patterns with conventional $x$-rays. The infection is often life-threatening and commonly seen in diabetic patients. Escherichia coli is the most common causative agent. A case is presented of emphysematous pyelonephritis, ureteritis, and cystitis which were aggressively treated with antibiotics and renal decompression by percutaneous nephrostomy. The clinical, pathologic, and management aspects of this often lethal infection are discussed.
\end{abstract}

A rare, often life-threatening infection of the kidneys, ureter, and bladder, with spontaneous generation of gas in these tissues, is known as emphysematous pyelonephritis, ureteritis, and cystitis. This disease entity usually occurs in diabetic patients, and the most common causative agent is a severe Escherichia coli infection involving the urinary tract. To date, 69 cases have been reviewed in the literature; one more case is added by our group. The radiographic, pathologic, and clinical manifestations of this suppurative infection are discussed.

\section{Report of case}

A 70-year-old Korean woman was admitted to the medical service for evaluation of left-sided abdominal pain and shortness of breath. She described her abdominal discomfort as dull and radiating to the left flank. There was no history of hematuria, pyuria, urinary frequency or urgency, or dysuria. The patient denied past calculus disease or upper tract infections. She was known to have steroid-dependent chronic obstructive pulmonary disease, for which she had been followed since her immigration in 1979.
Her initial temperature was $37.5^{\circ} \mathrm{C}$, but it climbed steadily to $39^{\circ} \mathrm{C}$. Other vital signs were as follows: blood pressure, $140 / 90 \mathrm{~mm} \mathrm{Hg}$; pulse rate, 100 beats per minute; and respiratory rate, 22 per minute. Abdominal palpation resulted in diffuse, left upper quadrant tenderness, and flank pain also was elicited. No rebound tenderness or rigidity was noted. The white blood cell count was $11,500 / \mathrm{cu} \mathrm{mm}$, with a differential count of $8 \%$ juvenile forms and $73 \%$ segmented neutrophils, significantly a left shift with $14 \%$ lymphocytes and $5 \%$ monocytes. Blood glucose was 110 . Other pertinent laboratory results were normal.

Gross hematuria was noted on the 14th hospital day, and urologic consultation was obtained. Intravenous pyelography revealed emphysematous cystitis, left ureterectasis and pyelitis, and high-grade left ureteral obstruction (Figs 1,2). Prior to genitourinary evaluation, the patient was started on IV hydration. Surgical and gynecologic consultations were obtained. Gas was demonstated throughout the left collecting system.

A percutaneous nephrostomy tube was placed to relieve the left ureteral obstruction. Urine was collected for routine culture and for sensitivity, acid-fast bacillus, fungi, and cytologic tests. Culture and the sensitivity test demonstrated $E$ coli; other cultures were negative. Cytologic testing revealed class cells.

Cystoscopy showed bullous changes in the mucosa of the urinary bladder, as well as hyperemia and hemorrhage. The ureteral orifices could not be identified, and bladder biopsy specimens showed cystitis cystica. Antegrade nephrostography demonstrated left ureteral obstruction at the pelvic brim. A Hippuran renal scan showed virtually no function of the left kidney, and scant urine volume was collected from the nephrostomy tube even after decompression.

Aggressive antimicrobial therapy, including a secondgeneration cephalosporin and an aminoglycoside for best aerobic gram-negative coverage, was started. Cultures of blood and urine were negative on the fifth day of antibiotic therapy. However, gas still was present on the plain abdominal film.

Subsequently, the patient underwent left nephrectomy. The pathologic report described microabscesses, with emphysematous pyelonephritis. Her postoperative course was unremarkable, her temperature defervesced, 
and she was discharged from the hospital on the tenth postoperative day.

\section{Discussion}

There is confusion regarding the proper nomenclature for gas-forming infections of the urinary tract. Terms such as renal emphysema, pneumonephritis, pyelonephritis emphysematosa, and emphysematous cystitis all have been used. Perhaps the best name is emphysematous pyelonephritis, because it describes an acute infection with gas production. Gas may be introduced into the urinary tract by three main mechanisms: (1) during procedures involving instrument use; (2) by fistula formation to a hollow viscus; and (3) by infectious agents. ${ }^{1}$ Gas may be found anywhere within the collecting system - from renal parenchyma to calices, from ureters to bladder.

In a comprehensive 1984 review of this disease entity, Michaeli and associates ${ }^{1}$ found 69 cases of emphysematous pyelonephritis in the literature, the first of which was described in 1898. According to Schultz and Klorfein, ${ }^{2}$ the average age of onset is 54 years, there is a female-male predominance, and the left kidney is most often involved.

\section{Diagnosis}

Gas-producing infections of the urinary tract are seen predominately in patients with diabetes; however, cases of nondiabetic patients with emphysematous pyelonephritis have been reported. ${ }^{3}$ Our patient also was free of diabetes. Emphysematous pyelonephritis may occur in the presence or absence of lower urinary tract obstruction. Klein and coauthors $^{4}$ stated that in nondiabetics, renal emphysema usually is secondary to a pre-existing urologic abnormality, such as congenital, inflammatory calculus or neoplastic obstruction involving some portion of the collecting system. We theorize that this overwhelming infection was caused by a lower tract infection that ascended to the kidney and ureters.

Clinical symptoms may be few, with only pyuria and bacteriuria noted; however, there often are complaints of nausea, vomiting, diarrhea, and dehydration. ${ }^{5}$ Patients often have chills and fever, flank pain, lethargy, and confusion. ${ }^{1}$ Pneumaturia usually is not seen in emphysematous pyelonephritis alone, but it will be present in emphysematous cystitis or when the two entities coexist. ${ }^{1,5}$ In many series, onset was sudden and symptoms progressed rapidly, ${ }^{5}$ but in their review, Michaeli and associates $^{1}$ found a more chronic nature to this disease (duration of two weeks to months).

Radiography is the best, quickest method for di-

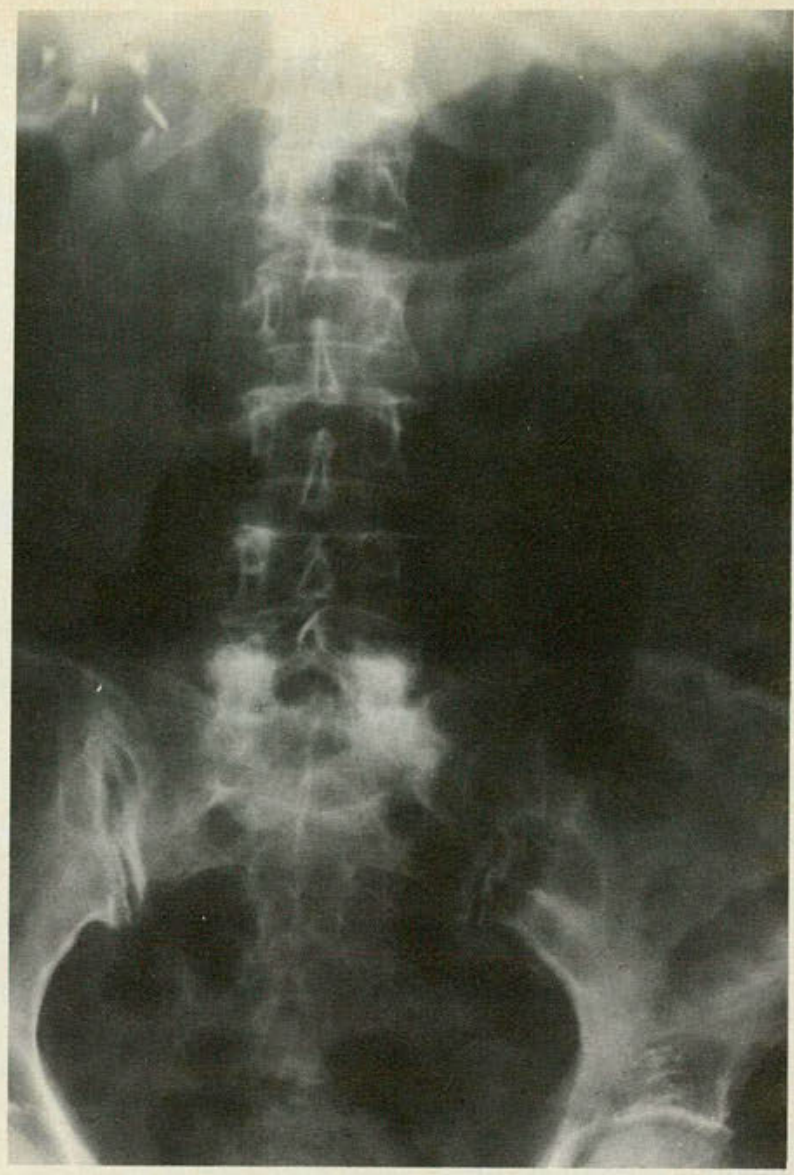

Fig 1. Survey abdominal $x$-ray demonstrates gas throughout the collecting system.

agnosing emphysematous pyelonephritis. ${ }^{1}$ Some difficulty has occurred with classification and description of the radiographic findings. Michaeli and associates ${ }^{1}$ proposed a simpler, less-descriptive classification: stage 1, presence of gas within the renal parenchyma; stage 2, presence of gas in the kidney and surrounding tissue; and stage 3 , bilateral involvement or extension of gas through Gerota's fascia.

The most common organism to produce gas in the urinary tract is $E$ coli, with Klebsiella pneumoniae, Aerobacter aerogenes, and Proteus mirabalis also isolated in some patients. ${ }^{1,4,5}$ In the diabetic patient, gas production can be attributed to glucose formation by Proteus species and most other coliform organisms. ${ }^{4}$

\section{Pathogenetic considerations}

The characteristic pathologic findings of emphysematous pyelonephritis are acute and chronic necrotizing pyelonephritis associated with multiple cor- 


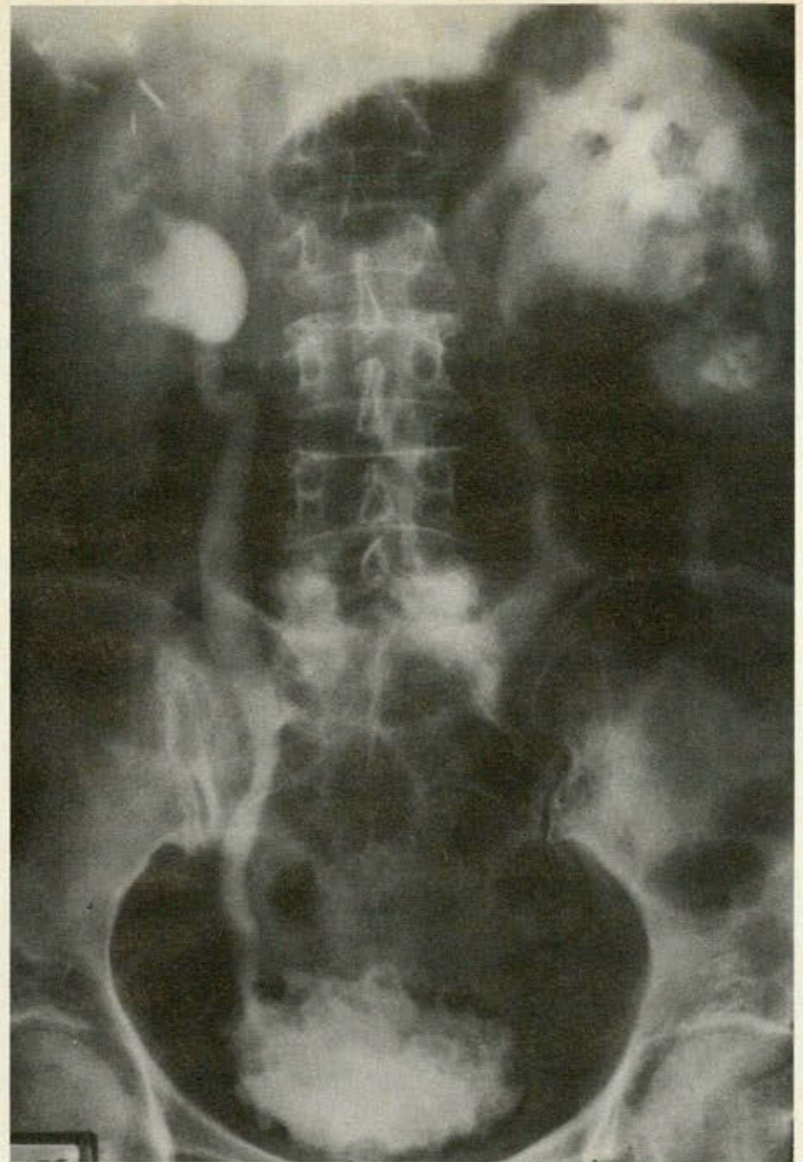

Fig 2. Intravenous pyelogram showing a high grade left ureteral obstruction.

tical abscesses..$^{1,2,6}$ Papillary necrosis and intrarenal vascular thrombi also have been reported. ${ }^{7,8}$

Many theories about the pathogenesis of this rare form of pyelonephritis exist. It has been postulated ${ }^{9}$ that the high urine and tissue concentration of glucose in diabetes mellitus patients provides infecting organisms with a substrate for fermentation and subsequent gas production. This theory falls short of explaining emphysematous pyelonephritis in the patient who is nondiabetic and the relative rarity of this presentation in patients with diabetes mellitus, who have a high frequency of bacterial infections.

Schainuck and colleagues ${ }^{10}$ theorize that impaired tissue and vascular responses are responsible for the clinical manifestations of emphysematous pyelonephritis, because the two important features of necrotizing infections and impaired vascular supply commonly are found. They further state that local factors (such as obstruction) and systemic factors (such as high risk of infections in persons with diabetes) contribute to tissue and vascular damage. It is the impaired host response, rather than hyperglycemia per se, that is responsible for gas production in the necrotic tissue. ${ }^{1}$ This impaired host response theory may be a good explanation for gas production in patients without diabetes mellitus or, possibly, those without evidence of infection. The coexistence of impaired host response and sugar fermentation may account for the production of large amounts of gas in diabetic patients.

\section{Treatment}

The therapeutic approach (conservative medical management $v$ aggressive surgical intervention) to patients with emphysematous pyelonephritis has been contested hotly. ${ }^{2,4}$ Michaeli and associates, ${ }^{1}$ in their review, found a sharp decrease in mortality rates from surgical and combined medical and surgical treatment over the past decade. They note that despite the widespread use of aminoglycosides, the fatality rate from antimicrobial treatment alone remains high. For the period 1970-1982, they report a $93 \%$ survival rate for patients who failed medical management but who underwent surgery quickly afterward. Because the host response may be compromised, antibiotic therapy with ampicillin, a second- or third-generation cephalosporin, and an aminoglycoside should be instituted immediately.

Michaeli and associates ${ }^{1}$ made several important conclusions regarding the management of emphysematous pyelonephritis, as follows: (1) Aggressive fluid resuscitation, control of shock, and treatment of diabetes mellitus must be initiated even before the diagnosis can be confirmed; (2) an attempt to ascertain the source of renal gas must be made; (3) antimicrobial therapy should be instituted as soon as diagnosis is made; (4) the obstructed system must be relieved; (5) the persistence of the intrarenal gas pattern and involvement of the contralateral side should be monitored; and (6) aggressive surgical intervention, even with bilateral involvement or even in solitary kidney disease, is recommended.

\section{Summary}

In the case reported here, the patient was a nondiabetic, 70-year-old woman. Urine cultures were positive for $E$ coli. The management regimen included decompression of ureteric obstruction with percutaneous nephrostomy tube insertion, aggressive antibiotic coverage, and subsequent nephrectomy. The patient made an uneventful recovery and was discharged from the hospital within three 
weeks of diagnosis and surgical intervention. Quick, accurate diagnosis, which is based on urine culture and characteristic radiography (including intravenous pyelography), combined with aggressive surgical and medical intervention enable the clinician to improve treatment outcomes in this previously highly morbid or fatal infection.

1. Michaeli J, Mogle P, Perlberg DS, et al: Emphysematous pyelonephritis. J Urol 1984;131:203-208.

2. Schultz EH Jr, Klorfein EH: Emphysematous pyelonephritis. J Urol 1962;87:762-766.

3. Sunanta A: Renal emphysema and cystitis emphysematosa: A case report. J Med Assoc Thai 1974;57:272-275.

4. Klein DE, Mahoney SA, Youngen R, et al: Renal emphysema $J$ Urol1966;95:625-629.

5. Stokes JB Jr: Emphysematous pyelonephritis. J Urol 1966;96:6-11.

6. Welch NM, Prather GC: Pneumonephrosis: A complication of necrotiz- ing pyelonephritis. $J$ Urol 1949;61:712-718.

7. Banks DE Jr, Persky L, Mahoney SA: Renal emphysema. J Urol 1969;102:390-395.

8. McMurray SD, Luft FC, Maxwell DR, et al: Emphysematous pyelonephritis. J Urol 1976;115:604-605.

9. Ireland GW, Javadpour N, Cass AS: Renal emphysema and retention of renal function $J$ Urol 1971;106:463-466.

10. Schainuck LI, Fouty R, Cutler RE: Emphysematous pyelonephritis: A new case and review of previous obervations. Am J Med 1968;44:134139.

From the Departments of Surgery and Radiology and Division of Urology, Albert Einstein Medical Center, Northern Division, Philadelphia.

Reprint requests to Dr Ginsberg, Suite 503, Klein Professional Office Bldg, 5401 Old York Rd, Philadelphia, PA 19141. 